\title{
Avoiding infinity in Quantum field theory without use of renormalization
}

\author{
Biswaranjan Dikshit \\ Laser and Plasma Technology Division \\ Bhabha Atomic Research Centre, Mumbai-400085, INDIA \\ Homi Bhabha National Institute, Mumbai-400094, INDIA \\ *Corresponding Author: bdikshit73@yahoo.co.in Tel.: +91-99696-17504
}

Available online at: www.isroset.org

Received: 16/Mar/2019, Accepted: 14/Apr/2019, Online: 30/Apr/2019

\begin{abstract}
In quantum field theory (QFT), it is well known that when Feynman diagrams containing loops are evaluated to account for self-interactions, probability amplitude comes out to be infinite which is physically not admissible. So, to make the QFT convergent, various renormalization methods are conventionally followed in which an additional (infinite) counter term is postulated which neutralizes the original infinity generated by diagram. The resulting finite values of amplitudes have agreed with experiments with surprising accuracy. However, proponents of renormalization methods acknowledged that this ad-hoc procedure of subtraction of infinity from infinity to reach at a finite value is not at all satisfactory and there is no physical basis for bringing in the counter term. So, it is desirable to establish a method in QFT which does not generate any infinite term, but which predicts same results as conventional methods do. In this paper, we describe such a technique taking self-interaction quantum electrodynamics diagram representing electron or photon self-energy. In our method, no problem of infinity arises and hence renormalization is not necessary. Still, the dependence of calculated probability amplitude on physical variables in our technique comes out to be same as conventional methods. Using similar procedure, we hope, the problem of nonrenormalizability of quantum gravity may be solved in future.
\end{abstract}

Keywords - Renormalization, Quantum Field Theory, Electron self energy, Vacuum polarization

\section{INTRODUCTION}

In a recent article [1] titled "There are no particles, only fields", Hobson has clearly explained the stand taken by quantum field theorists that all physical objects in the universe are basically fields (waves). Light and matter both exist as waves while their interaction takes place at points in space (as in photoelectric effect, Compton scattering etc.). For example, an electron is actually a quantum of electron field which is represented by Dirac equation. Hence, contrary to classical point model of electron in which self-energy becomes infinity, quantum field theory (QFT) was expected to be convergent as in this case electron field is spread out in space. However, Feynman calculations of self-interaction diagrams of electrons and photons were found to be divergent. This resulted in stagnancy of QFT for almost two decades after 1930. Then came the innovative idea of renormalization to tackle the infinity arising in calculations and to make experimentally verifiable predictions [2-6]. Renormalization involves two steps, regularization and then renormalization. In regularization, the divergent part in mathematical expression of probability amplitude is isolated from the rest. For instance, in cut off regularization [2], upper limit of four momentum of virtual particle is taken as
$\Lambda$ instead of $\infty$ so that algebraic manipulations are possible. However, at the end when $\Lambda$ is raised to its real value $\infty$, the term containing $\Lambda$ in probability amplitude also becomes infinity. Similarly, in the famous dimensional regularization scheme, all calculations are carried out in (4- $\varepsilon$ ) space-time dimensions and finally $\varepsilon$ is taken to zero for predictions in real world of 4-dimensions. For example, in case of a oneloop self-interaction diagram as given in Fig. 1, probability amplitude in 4-dimensions derived by Griffiths [7] (taking simplified assumptions of zero spin) is given by,

$$
I=-\left[\operatorname{Lim}_{\varepsilon \rightarrow 0}\left(\frac{g}{4 \pi}\right)^{2} \frac{2}{\varepsilon}\right]+\left(\frac{g}{4 \pi}\right)^{2}\left[\gamma+\int_{0}^{1} \ln \left(\frac{N^{2}}{4 \pi(M c)^{2}}\right) d x\right]
$$

Where, $g$ is experimentally observed coupling constant in 4dimensions and it is related to dimensionless coupling constant $g_{0}$ by, $g=g_{0} M c$. Here, $M$ is an arbitrary constant having dimensions of mass which needs to be determined from experiment and $c$ is speed of light. The variable $N^{2}$ is given by,

$$
N^{2}=\bar{p}^{2} x(1-x)+m_{B}^{2} c^{2}(1-x)+m_{C}^{2} c^{2} x
$$

where, $\quad \bar{p}^{2}=-\left(p_{0}\right)^{2}+\left(p_{1}\right)^{2}+\left(p_{2}\right)^{2}+\left(p_{3}\right)^{2}$ 
and $p_{0}, p_{1}, p_{2}, p_{3}$ are components of four momentum of incoming particle A. The value of $\gamma$ is $0.577 \ldots$ (EulerMascheroni constant). Note that as soon as limit $(\varepsilon=0)$ is put, first term in Eq. (1) blows up to infinity. This was a catastrophe in the history of quantum field theory. To rescue us from this impasse, innovative idea of renormalization scheme was brought in. In this procedure, an additional selfinteraction diagram (or counter term) with a cross in place of loop was introduced whose amplitude exactly cancels the divergent part in Eq. (1) so that net amplitude remains finite. Naturally, the magnitude of counter term is also infinity. In other words, we have simply subtracted infinity from Eq. (1) to avoid unwanted divergence. Although we can say that infinite counter term is already included in observed mass, there is no physical justification for this. The proposal that infinite mass counter term might be coming from general relativistic effects [8-9] is not acceptable due to following reason. If QFT is true, then so called particles are actually fields (or waves) spread out in space-time. So, their small masses will not be sufficient to warp space-time to an extent generating infinite gravitational energy. We also cannot escape from the inconsistency of mathematical expressions on the pretext of ignorance of high energy behavior of particles especially when QFT is itself made for relativistic particles. Recently, Altaisky [10] proposed to take into account the resolution of the measuring instrument to make the QFT results finite. But, in our view, QFT must be made mathematically consistent irrespective of our measuring capability. Claims of reproducing QFT results using classical field theory without field quantization [11-12] has also been questioned by Bialynicki-Birula [13] and we cannot forgo field quantization as it is today a firmly and experimentally established fact.

Thus, the ad-hoc procedure of renormalization, although helps us in calculation of observable parameters, is not at all intellectually satisfying. That's why, one of the founders of QED, Dirac had stated [14],

"Most physicists are very satisfied with the situation.

They say: 'Quantum electrodynamics is a good theory and we do not have to worry about it anymore.' I must say that I am very dissatisfied with the situation, because this so-called 'good theory' does involve neglecting infinities which appear in its equations, neglecting them in an arbitrary way. This is just not sensible mathematics. Sensible mathematics involves neglecting a quantity when it is small - not neglecting it just because it is infinitely great and you do not want it!"

Similarly, Feynman, who played a crucial role in development of quantum electrodynamics wrote [15] in 1985 ,

"The shell game that we play ... is technically called 'renormalization'. But no matter how clever the word, it is still what I would call a dippy process! Having to resort to such hocus-pocus has prevented us from proving that the theory of quantum electrodynamics is mathematically self-consistent. It's surprising that the theory still hasn't been proved self-consistent one way or the other by now; I suspect that renormalization is not mathematically legitimate".

So, now it is very important that we look for a new approach in QFT calculations which does not produce the infinite term (hence, not requiring renormalization), but reproduces the same finite experimentally verifiable result as conventional methods do. In this paper, we describe such an approach in quantum electrodynamics for a single loop Feynman diagram in which no infinite term appears in the final expression of probability amplitude and thus, renormalization is not required. Dependence of the measurable parameter (probability amplitude) on the input variables in our approach is found to be same as in the conventional expression given by Eq. (1).

\section{NEW APPROACH FOR QED CALCULATION WITHOUT RENORMALIZATION}

Consider a self-interaction Feynman diagram containing a single loop as given in Fig.1. In this diagram, incoming particle A with four momentum $p$ splits into two virtual particles $\mathrm{B}$ and $\mathrm{C}$, and finally $\mathrm{A}$ is created again by recombination. Four momentum of virtual particle $\mathrm{B}$ is $q$.

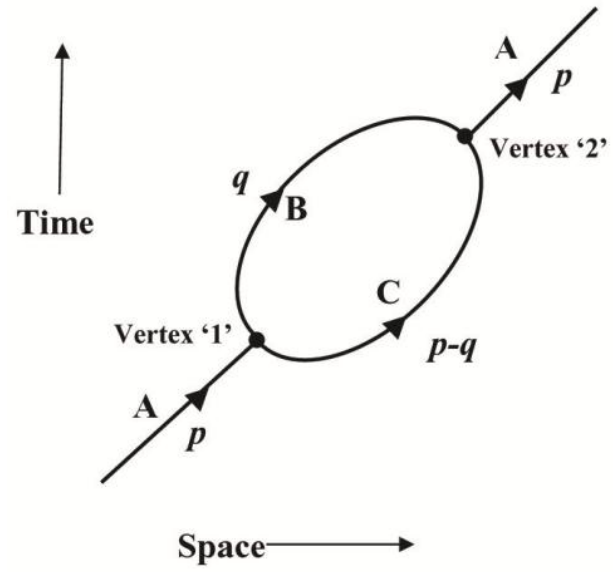

Figure 1. Single loop Feynman diagram for self-interaction (Electron self-energy or vacuum polarization)

So, by energy-momentum conservation, four momentum of $\mathrm{C}$ is $(p-q)$. This diagram can represent either electron selfenergy or vacuum polarization by selecting appropriate masses for particles. Ignoring spin for simplicity, probability amplitude contributed by the diagram is given by (see Griffiths [7]),

$$
I=\frac{i g^{2}}{(2 \pi)^{4}} \int \frac{d^{4} q}{\left(q^{2}-m_{B}^{2} c^{2}\right)\left[(p-q)^{2}-m_{C}^{2} c^{2}\right]}
$$


After using Wick rotation and Feynman trick as illustrated by Griffiths [7], Eq. (2) reduces to,

$I=-\frac{g^{2}}{(2 \pi)^{4}} \times$

$\int_{x=0}^{1}\left[\int_{-\infty}^{\infty} \int_{-\infty}^{\infty} \int_{-\infty-\infty}^{\infty} \int_{0}^{\infty} \frac{d q_{1}^{\prime} d q_{2}^{\prime} d q_{3}^{\prime} d q_{4}^{\prime}}{\left[\left(q_{1}^{\prime}\right)^{2}+\left(q_{2}^{\prime}\right)^{2}+\left(q_{3}^{\prime}\right)^{2}+\left(q_{4}^{\prime}\right)^{2}+N^{2}\right]^{2}}\right] d x$

Where $\quad q_{1}^{\prime}=q_{1}-p_{1} x, q_{2}^{\prime}=q_{2}-p_{2} x$,

$$
q_{3}^{\prime}=q_{3}-p_{3} x, q_{4}^{\prime}=q_{4}-p_{4} x
$$$$
q_{4}=-i q_{0} \text { and } p_{4}=-i p_{0} \text {. }
$$

$p_{0}, p_{1}, p_{2}, p_{3}$ are components of four momentum $p$ and $q_{0}, q_{1}, q_{2}, q_{3}$ are components of $q$. The mathematical parameter $x$ is dimensionless and varies from 0 to 1 .

The variable $N^{2}$ is given by,

$$
N^{2}=\bar{p}^{2} x(1-x)+m_{B}^{2} c^{2}(1-x)+m_{C}^{2} c^{2} x
$$

where, $\quad \bar{p}^{2}=\left(p_{1}\right)^{2}+\left(p_{2}\right)^{2}+\left(p_{3}\right)^{2}+\left(p_{4}\right)^{2}$

If $A$ is a real particle,

$\bar{p}^{2}=\left(p_{1}\right)^{2}+\left(p_{2}\right)^{2}+\left(p_{3}\right)^{2}-\left(p_{0}\right)^{2}=-m_{A}^{2} c^{2}$.

Then, $N^{2}=m_{B}^{2} c^{2}(1-x)+m_{C}^{2} c^{2} x-m_{A}^{2} c^{2} x(1-x)$

Now, let us discuss two new assumptions that will be used in our formulation.

a) The problem of infinity in QFT has been existing since 1950 when there was a hope of hidden variables (as described by EPR paradox [16]) and concept of "undefined or superposition state" was not fully accepted. But, now quantum entanglement has already experimentally established that spin of entangled pair of photons emitted from a spin zero (singlet) atom remains in undefined state until it is measured along a direction (i.e. until a direction is defined by measuring instrument). Now, we all agree that physical property can be in a "undefined state". So, we will take the clue from this quantum entanglement to assume that momentum of virtual particles perpendicular to plane containing momentum and displacement of parent particle from which they are created are undefined. In other words, virtual particles cannot discover a new direction in space which their parents didn't describe. Hence, this undefined momentum should not be considered in integration to calculate probability amplitude. Thus, our method incorporates a hypothesis based on recent experimental observations so that problem of infinity doesn't appear in QFT. We think, this is a reasonable assumption as compared to the conventional method of renormalization where infinite term is neutralized by assuming a negative infinite counter term in the measured physical parameter (such as mass). Now coming to Fig. 1, virtual particles $\mathrm{B}$ and $\mathrm{C}$ represent the unobservable intermediate states in the form of which the parent particle $\mathrm{A}$ is displaced from vertex 1 to 2 . The parent particle A describes only two directions in space, one by its own momentum vector and other by its displacement vector from vertex 1 to 2 . Now, using the above described hypothesis, if the two-dimensional plane containing 3momentum and displacement of the parent particle is named as $\mathrm{X}$-Y plane (so that $p_{3}=0$ ), then the momentum of two virtual particles will always lie on this $\mathrm{X}-\mathrm{Y}$ plane. As virtual particles have no freedom for momentum along $Z$ direction due to lack of definiteness, in Eq. (3) we should not integrate with respect to $q_{3}^{\prime}$.

b) It is known that virtual particles are off-shell, meaning they need not satisfy Einstein's energy-momentum-mass relation $\left(E^{2} \neq p^{2} c^{2}+m^{2} c^{4}\right.$ ). In addition to this, in our approach, we will assume that masses of virtual particles have some amount of flexibility. For example, $m_{B}$ can be $m_{B}^{\prime}$ and $m_{C}$ can be $m_{C}^{\prime}$. Thus, $N$ in Eq. (5) becomes a variable which we will call as $N^{\prime}$. Since $N$ has the dimensions of momentum and it is now a variable $N^{\prime}$ depending upon masses of virtual particles, in Eq. (3) we should integrate with respect to $N^{\prime}$ to include all possibilities in the total amplitude. Limits for magnitude of $N^{\prime}$ will be from $N$ to some arbitrary constant $k$ which needs to be determined from experiment, just as in conventional method of QED, constant $M c$ in Eq. (1) is experimentally determined. As evident from Eq. (5), $N^{\prime}$ can be of both signs $(+$ or - ). So, we have to multiply the final expression of amplitude by a factor of ' 2 ' to account for both the possibilities.

Now using the above two assumptions, expression for probability amplitude in Eq. (3) is modified as,

$$
\begin{aligned}
& I=-\frac{g^{2}}{(2 \pi)^{4}} \times \\
& \int_{x=0}^{1} 2\left[\int_{N}^{k} \int_{-\infty}^{\infty} \int_{-\infty-\infty}^{\infty} \int_{-\infty}^{\infty} \frac{\left.\left.d q_{1}^{\prime} d q_{2}^{\prime} d q_{4}^{\prime} d N^{\prime}\right)^{2}+\left(q_{2}^{\prime}\right)^{2}+\left(q_{4}^{\prime}\right)^{2}+N^{\prime 2}\right]^{2}}{\left[\left(q_{1}\right.\right.}\right] d x
\end{aligned}
$$

The integration with respect to $q_{1}^{\prime}, q_{2}^{\prime}$ and $q_{4}^{\prime}$ can be carried out in spherical coordinates and the result is,

$$
\int_{-\infty}^{\infty} \int_{-\infty}^{\infty} \int_{-\infty}^{\infty} \frac{d q_{1}^{\prime} d q_{2}^{\prime} d q_{4}^{\prime}}{\left[\left(q_{1}^{\prime}\right)^{2}+\left(q_{2}^{\prime}\right)^{2}+\left(q_{4}^{\prime}\right)^{2}+N^{\prime 2}\right]^{2}}=\frac{\pi^{2}}{N^{\prime}}
$$

So, Eq. (6) becomes,

$$
I=-\frac{g^{2}}{(2 \pi)^{4}} \int_{x=0}^{1} 2 \pi^{2}\left[\int_{N}^{k} \frac{d N^{\prime}}{N^{\prime}}\right] d x
$$




$$
\text { Or } \quad I=\left(\frac{g}{4 \pi}\right)^{2} \int_{0}^{1} \ln \left(\frac{N}{k}\right)^{2} d x
$$

Thus, we see that dependence of amplitude $I$ on $N$ in our approach comes out to be same as in conventional method given by Eq. (1). If we wish, we can directly use Eq. (8) for calculating amplitude after finding the value of constant ' $k$ ' from experimental data. However, if we want the final expression exactly in the conventional form, we have to proceed further as given below.

As ' $k$ ' is an arbitrary constant which needs to be determined from experiment, we can write it in terms of arbitrary constant $M c$ of conventional method (which is also fixed by experiment) by the relation,

$$
k=\sqrt{4 \pi} M c e^{\frac{-\gamma}{2}}
$$

(where $\gamma=0.577 \ldots$ is Euler-Mascheroni constant)

This is due to the fact that physics remains same irrespective of whether we directly fit Eq. (8) on experimental data to find $k$ or we first put Eq. (9) in Eq. (8) and then fit experimental data to find $M c$. So, putting Eq. (9) in Eq. (8),

$$
\begin{aligned}
I & =\left(\frac{g}{4 \pi}\right)^{2} \int_{0}^{1} \ln \left(\frac{N}{\sqrt{4 \pi} M c e^{\frac{-\gamma}{2}}}\right)^{2} d x \\
\text { Or } \quad I & =\left(\frac{g}{4 \pi}\right)^{2} \int_{0}^{1}\left[\ln e^{\gamma}+\ln \left(\frac{N}{\sqrt{4 \pi} M c}\right)^{2}\right] d x \\
\text { Or } \quad I & =\left(\frac{g}{4 \pi}\right)^{2}\left[\gamma+\int_{0}^{1} \ln \left(\frac{N^{2}}{4 \pi(M c)^{2}}\right) d x\right]
\end{aligned}
$$

We can check that Eq. (10) derived in our method is exactly same as the finite part of Eq. (1) of conventional method which has been experimentally established. Since in our method, infinite term does not arise, we don't need renormalization which is an arbitrary process of neglecting infinity to get a finite answer.

\section{CONCLUSION}

In this paper, we have proved that the probability amplitude for self-interaction (electron self-energy or vacuum polarization) represented by a single loop Feynman diagram can be exactly derived matching with the finite term of conventional method that agrees with experiment, but without producing any associated infinite term. Hence, in our approach, we don't need renormalization which is used in conventional approach of quantum field theory to neglect the infinity and which has been doubted as an illegitimate mathematical procedure even by proponents of QFT such as Dirac and Feynman. We could escape from the long-standing problem of divergence (infinity) by assuming that momentum of virtual particles along a direction perpendicular to two-dimensional plane described by their parent particle remains undefined. This hypothesis was motivated from the observation that spin of two entangled particles remains undefined until it is measured. Using similar technique, we hope, the problem of nonrenormalizability of quantum gravity may be solved in future.

\section{ACKNOWLEDGMENT}

Author is grateful to Mr. R K Rajawat, Associate Director of Beam Technology Development Group, Bhabha Atomic Research Centre for his encouragement and support during this research.

\section{REFERENCES}

[1] Art Hobson, "There are no particles, there are only fields", American Journal of Physics, Vol. 81(3), pp. 211-223, 2013

[2] David Griffiths, "Introduction to Elementary particles, (WileyVCH Verlag, Weinheim), 2008

[3] $\mathrm{G}$ 't Hooft and $\mathrm{M}$ Veltman, "Regularization and Renormalization of gauge fields", Nuclear Physics B, Vol. 44, pp. 189-213, 1972

[4] R P Feynman, Quantum Electrodynamics: A lecture note and reprint volume, $\mathrm{W}$ A Benjamin Inc, New York, 1961

[5] M E Peskin and D V Schroeder, An Introduction to quantum field theory", Addison-Wesley, Massachusetts, 1995

[6] A Zee, Quantum Field Theory in a Nutshell, Princeton University press, New Jersey, 2010

[7] Per Kraus and David J Griffiths, "Renormalization of a model quantum field theory", American Journal of Physics, Vol. 60 (11), pp. 1013-1023, 1992

[8] C J Isham, Abdus Salam and J Strathdee, "Infinity suppression in Gravity-modified Quantum Electrodynamics", Physical Review D, Vol. 3(8), pp. 1805-1817, 1971

[9] I E Segal and Z Zhou, "Convergence of Quantum Electrodynamics in a Curved Deformation of Minkowski space", Annals of Physics, Vol. 232, pp. 61-87, 1994

[10] M V Altaisky, "Quantum field theory without divergences", Physical Review D, Vol. 81, 125003, 2010

[11] A O Barut and J F Van Huele, "Quantum electrodynamics based on self-energy: Lamb shift and spontaneous emission without field quantization", Physical Review A, Vol. 32 (6), pp. 3187-3195, 1985

[12] I Acikgoz, A O Barut, J Kraus and N Unal, "Self-field quantum electrodynamics without infinities: A new calculation of vacuum polarization", Physics letters A, Vol. 198, pp. 126130, 1995

[13] Iwo Bialynicki-Birula, "Comment on "Quantum electrodynamics based on self-energy: Lamb shift and spontaneous emission without field quantization", Physical review A, Vol. 34 (4), pp. 3500-3501, 1986 
[14] Kragh Helge, Dirac: A scientific biography, (Cambridge University Press, New York), 1990, p. 184

[15] R P Feynman, QED, The Strange Theory of Light and Matter, Penguin, London, p. 128, 1990,

[16] A Einstein, B Podolsky and N Rosen, "Can QuantumMechanical Description of Physical reality be considered complete?", Physical Review, Vol. 47, pp. 777-780, 1935

\section{AUTHORS PROFILE}

Dr Biswaranjan Dikshit received his Ph.D. degree from Homi Bhabha National Institute, Mumbai, India in 2011. He is currently working as Scientific Officer in Laser and Plasma Technology Division of Bhabha Atomic Research Centre, Mumbai and is recognized as a Professor by Homi Bhabha National Institute, Mumbai. He has 24 years of

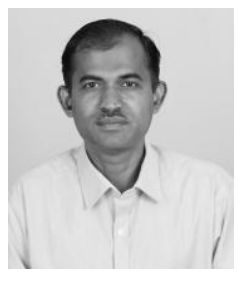
research experience and published more than 28 papers in international journals. His current fields of interest are Electrodynamics, Relativity and Quantum physics. 